\title{
Reverse rate-dependent changes are determined by baseline action potential duration in mammalian and human ventricular preparations
}

\author{
László Bárándi • László Virág • Norbert Jost • Zoltán Horváth • István Koncz • \\ Rita Papp · Gábor Harmati · Balázs Horváth · Norbert Szentandrássy · Tamás Bányász • \\ János Magyar · Antonio Zaza · András Varró · Péter P. Nánási
}

Received: 30 November 2009/Revised: 10 December 2009/Accepted: 21 December 2009/Published online: 3 February 2010

(C) Springer-Verlag 2010

\begin{abstract}
Class III antiarrhythmic agents exhibit reverse rate-dependent lengthening of the action potential duration (APD). In spite of the several theories developed so far to explain this reverse rate-dependency (RRD), its mechanism has not yet been clarified. The aim of the present work was to further elucidate the mechanisms responsible for RRD in mammalian ventricular myocardium. Action potentials were recorded using conventional sharp microelectrodes from human, canine, rabbit and guinea pig ventricular myocardium in a rate-dependent manner varying the cycle length (CL) between 0.3 and $5 \mathrm{~s}$. Rate-dependent drug effects were studied using agents known to lengthen or shorten action potentials, and these drug-induced changes in APD were correlated with baseline APD values. Both drug-induced lengthening (by dofetilide, sotalol, E-4031, $\mathrm{BaCl}_{2}$, veratrine, BAY K 8644) and shortening (by mexiletine, tetrodotoxin, lemakalim) of action potentials displayed RRD, i.e., changes in APD were greater at longer than at
\end{abstract}

L. Bárándi · G. Harmati · B. Horváth · N. Szentandrássy ·

T. Bányász · J. Magyar · P. P. Nánási (\)

Department of Physiology, University of Debrecen,

Nagyerdei krt 98, 4012 Debrecen, Hungary

e-mail: nanasi@phys.dote.hu

L. Virág · I. Koncz · R. Papp · A. Varró

Department of Pharmacology and Pharmacotherapy,

University of Szeged, Szeged, Hungary

N. Jost · Z. Horváth · A. Varró

Division of Cardiovascular Pharmacology,

Hungarian Academy of Sciences, Szeged, Hungary

A. Zaza

Dipartimento di Biotecnologie e Bioscienze,

Universita di Milano-Bicocca, Milan, Italy shorter CLs. In rabbit, where APD is a biphasic function of $\mathrm{CL}$, the drug-induced APD changes were proportional to baseline APD values but not to CL. Similar results were obtained when repolarization was modified by injection of inward or outward current pulses in isolated canine cardiomyocytes. In each case the change in APD was proportional to baseline APD (i.e., that measured before the superfusion of drug or injection of current). Also, the net membrane current $\left(I_{\text {net }}\right)$, determined from the action potential waveform at the middle of the plateau, was inversely proportional to APD and consequently with to CL. The results indicate that RRD is a common characteristic of all the drugs tested regardless of the modified ion current species. Thus, drug-induced RRD can be considered as an intrinsic property of cardiac membranes based on the inverse relationship between $I_{\text {net }}$ and APD.

Keywords Ventricular repolarization - Action potential duration - Reverse rate dependence - Membrane current . Human myocardium $\cdot$ Mammalian cardiac cells

\section{Introduction}

Class III antiarrhythmic agents are known to display a reverse rate-dependent lengthening of APD, called RRD $[6,9]$. This RRD nature of the APD lengthening is undesirable because it minimizes drug effects on repolarization during tachyarrhythmias and enhances their proarrhythmic potential at normal rates [6, 11, 18]. Several hypotheses have been developed so far to explain the mechanism of RRD. Based on results obtained in guinea pig ventricular myocytes, it was first suggested that significant accumulation of $I_{\mathrm{Ks}}$ may occur due to the 
incomplete deactivation of the current at fast heart rates, which would greatly attenuate the APD lengthening effect of $I_{\mathrm{Kr}}$ blockade [9]. According to another hypothesis the effect of $I_{\mathrm{Kr}}$ blockers is reduced, and consequently the drug-induced prolongation of APD becomes diminished, at fast heart rates due to the potassium accumulation in the sarcolemmal clefts. This theory was based on observations showing that $I_{\mathrm{Kr}}$ blockade by quinidine and dofetilide was attenuated when extracellular potassium concentration was elevated [19]. A third hypothesis, the modulated receptor theory, was based on the rate dependency of drug-channel interactions stating that drug binding and unbinding are strongly dependent on the state of the channel, which is dynamically changing during the cardiac cycle $[4,5]$. Assuming that $I_{\mathrm{Kr}}$ blockers may be binding preferably during the diastole and unbinding during the action potential, RRD can be expected indeed. Finally, rate-dependent changes in action potential morphology were also claimed to contribute to the reverse rate dependency by controlling the kinetic properties of $I_{\mathrm{Kr}}$ and $I_{\mathrm{K} 1}$ in guinea pig, dog, and human ventricular preparations $[12,16]$.

The multitude of theories suggested explaining RRD indicates that its exact mechanism is still unclear. In a recent study in dog myocardium [1], APD modulation by drugs with diverse mechanisms was invariably characterized by RRD. This was interpreted as RRD being an "intrinsic" property, resulting from the shape of the relationship between $I_{\text {net }}$ and APD $[1,20]$. Such an interpretation was based on the assumption that APD modulation depends on drug-induced changes in $I_{\text {net }}$, independently of the specific current affected. The interpretation also predicts that, instead of depending on heart rate (as suggested by the term RRD), the extent of drug-induced APD change may be a function of baseline APD, the latter being in turn a function of rate.

The aims of the present study were to test (1) whether RRD is a general property of APD modulation also in other species, including man; (2) the assumption and the predictions of the interpretation of RRD provided in our previous work $[1,20]$.

\section{Methods}

\section{Animal tissue preparations}

Adult mongrel dogs (10-15 kg), guinea pigs $(0.3-0.5 \mathrm{~kg})$, and New Zealand white rabbits $(2-3 \mathrm{~kg})$ of either sex were anesthetized using sodium pentobarbital. After opening the chest the hearts were rapidly removed and

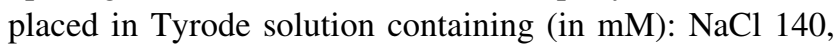
$\mathrm{KCl} 5.4, \mathrm{CaCl}_{2}$ 2.5, $\mathrm{MgCl}_{2}$ 1.2, HEPES 5, glucose 10, at
pH 7.4. Papillary muscles, excised from both ventricles, were used for standard microelectrode measurements. A wedge-shaped section of the left ventricular wall of canine hearts, supplied by the left anterior descending coronary artery, was dissected and cannulated for isolation of single myocytes. All experiments were carried out in compliance with the Guide for the Care and Use of Laboratory Animals (USA NIH publication NO 86-23, revised 1985), and the protocols were approved by the local ethical committee (CAR I-74-66/2005). Chemicals used in the experiments were obtained from SigmaAldrich Co. (St. Louis, MO, USA).

Human tissue preparations

Papillary muscles were excised also from undiseased donor hearts. The hearts were obtained from general organ donors, their valves were utilized for pulmonary and aortic valve transplantation surgery. Before explantation of the hearts the patients did not receive any medication except for dobutamine, furosemide and plasma expanders. The experimental protocol complied with the ethical standards laid down in the 1964 Declaration of Helsinki and was approved by the Ethical Review Board of the Albert Szent-Györgyi Medical University (No. 5157/1997 OEJ).

Action potential recording in multicellular muscle preparations

Papillary muscles were mounted in a plexiglass chamber, allowing continuous superfusion with oxygenized Tyrode solution at $37^{\circ} \mathrm{C}$, and paced through a pair of platinum electrodes. The pacing cycle length was initially set to $1 \mathrm{~s}$ for at least $60 \mathrm{~min}$ allowing the preparations to equilibrate before starting the measurement. Following equilibration the cycle length was set to $5 \mathrm{~s}$, then it was gradually reduced to $3,2,1.5,1,0.7,0.5,0.4$, and $0.3 \mathrm{~s}$ at least for $5 \mathrm{~min}$ at each cycle length. This period of time was sufficient for stabilization of action potential parameters, including APD. After taking control records the preparations were superfused for $40 \mathrm{~min}$ with the drug tested and the measurement was repeated at each pacing cycle length. Glass microelectrodes, filled with $3 \mathrm{M} \mathrm{KCl}$ and having tip resistances of 5-20 M $\Omega$, were used to record transmembrane potentials after amplification (Experimetria Ltd., Budapest, Hungary). Action potential duration at $90 \%$ of depolarization $\left(\mathrm{APD}_{90}\right)$ was determined automatically by a HSE-APES software running on an IBM compatible computer containing an ADA 3300 analogue-to-digital data acquisition board (Real Time Devices Inc., State College, PA, USA) with a 
Fig. 1 a-f Cycle lengthdependent changes of action potential duration $\left(\mathrm{APD}_{90}\right)$ induced by $50 \mathrm{nM}$ dofetilide (a, $n=5), 30 \mu \mathrm{M}$ sotalol (b, $n=4), 1 \mu \mathrm{M} \mathrm{E}-4031$ (c, $n=6), 10 \mu \mathrm{M} \mathrm{BaCl} 2$ (d, $n=5), 10 \mu \mathrm{M}$ mexiletine (e, $n=4)$, and $2 \mu \mathrm{M}$ tetrodotoxin $(\mathbf{f}, n=3)$ in human papillary muscle preparations. The pacing cycle length was gradually decreased from 5 to $0.3 \mathrm{~s}$ ) before and $40 \mathrm{~min}$ after administration of drugs. g, h Drug-induced changes, plotted against baseline (pre-drug) APD values. APD changes in panel $\mathbf{g}$ are displayed as absolute magnitudes, while in $\mathbf{h}$ in a percentage form. Symbols and bars denote mean \pm SEM values
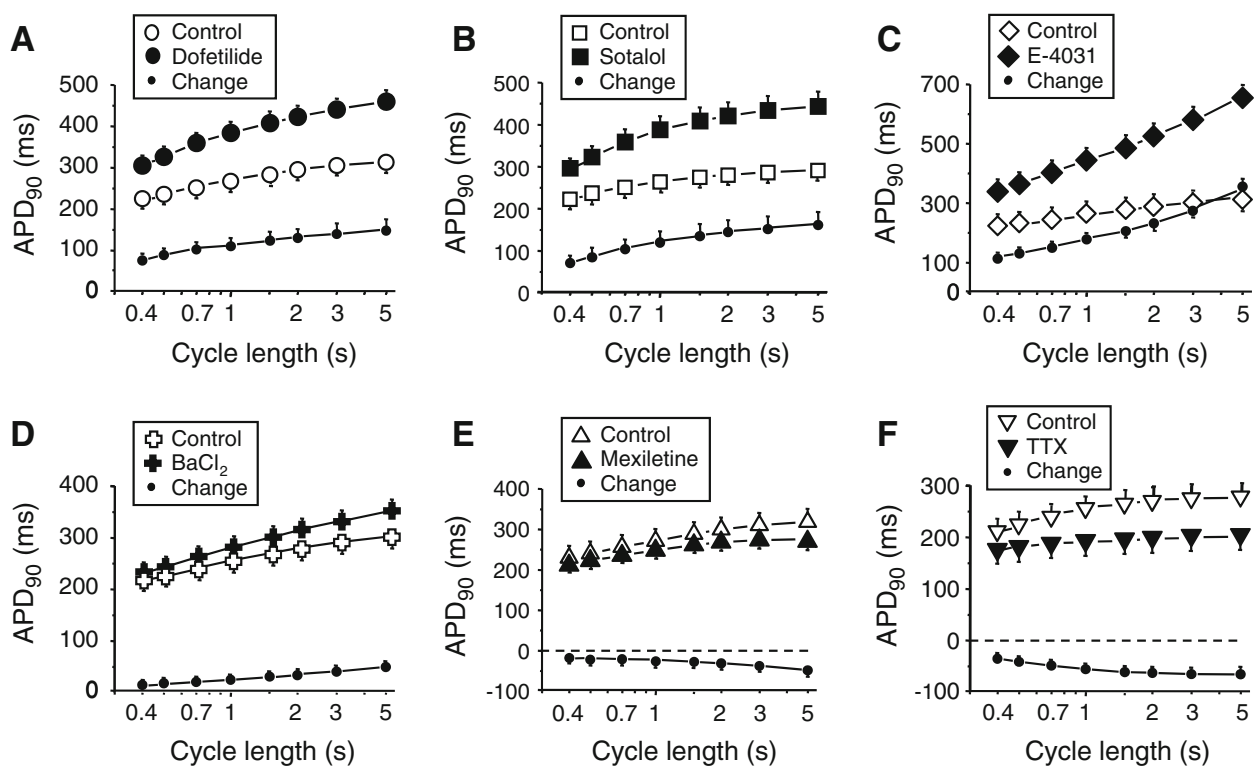

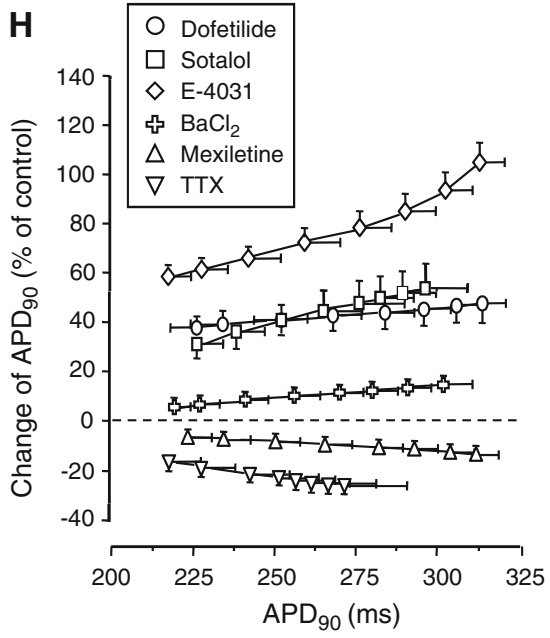

maximum sampling rate of $40 \mathrm{kHz}$. Efforts were made to maintain the same impalement throughout each experiment. When the impalement became dislodged, adjustment was attempted, and if the action potential characteristics of the re-established impalement deviated by less than $5 \%$ from the previous measurement the experiment continued.

Action potential recordings in isolated cardiomyocytes

Single canine ventricular myocytes were obtained by enzymatic dispersion using the segment perfusion technique [10]. The cells were sedimented in a plexiglass chamber and superfused with oxygenized Tyrode solution at $37^{\circ} \mathrm{C}$. Only rod shaped cells with clear cross striations were used. Transmembrane potentials were recorded using
$3 \mathrm{M} \mathrm{KCl}$ filled sharp glass microelectrodes having tip resistance between 20 and $40 \mathrm{M} \Omega$. These electrodes were connected to the input of an Axopatch-200B amplifier (Axon Instruments, Foster City, CA, USA) under current clamp conditions, allowing for simultaneous recording of action potentials and injection of inward or outward current pulses. These current pulses (having amplitudes of either -30 or $+60 \mathrm{pA}$, respectively, and durations longer than the expected APD) were injected from the upstroke of every 20th action potential at any cycle length tested (following the 5-min period of equilibration at the given cycle length, as described above). Thus, action potentials with current injections were separated from one another by 19 regular action potential waveforms. The cells were paced through the recording electrode using 1-ms-wide rectangular current pulses with twice threshold amplitude. The cycle 
length was initially set to $5 \mathrm{~s}$, and following stabilization of action potential configuration the cycle length was continuously varied to shorter values. Since the cytosol was not dialyzed, time-dependent changes in action potential duration were negligible for at least $60 \mathrm{~min}$ under these experimental conditions [7].

Estimation of net transmembrane current density

$I_{\text {net }}$ (in $\mathrm{pA} / \mathrm{pF}$ ) was calculated from the first derivative of membrane potential changes during repolarization
$\left(I_{\text {net }}\right.$ density $\left.=-\mathrm{d} V / \mathrm{d} t\right) . I_{\text {net }}$ was obtained at half duration of the action potential by considering a 50-ms-long segment of repolarization (half AP duration $\pm 25 \mathrm{~ms}$ ).

\section{Statistics}

Results are expressed as mean \pm SEM values. Statistical differences were evaluated using ANOVA. Differences were considered significant when $P$ was less than 0.05 .
Fig. 2 a-d Cycle lengthdependent changes in APD induced by $10 \mu \mathrm{M} \mathrm{BaCl} 2$ (a, $n=11), 1 \mu \mathrm{M}$ dofetilide (b, $n=13), 1 \mu$ M BAY K 8644 (c, $n=6$ ), and $1 \mu \mathrm{g} / \mathrm{ml}$ veratrine $(\mathbf{d}, n=7)$ in canine papillary muscles. The pacing cycle length was gradually decreased from 5 to $0.3 \mathrm{~s}$ ) before and $40 \mathrm{~min}$ after administration of drugs. Druginduced changes, plotted against baseline (pre-drug) APD values, are summarized in panel e. Symbols and bars denote means \pm SEM
A

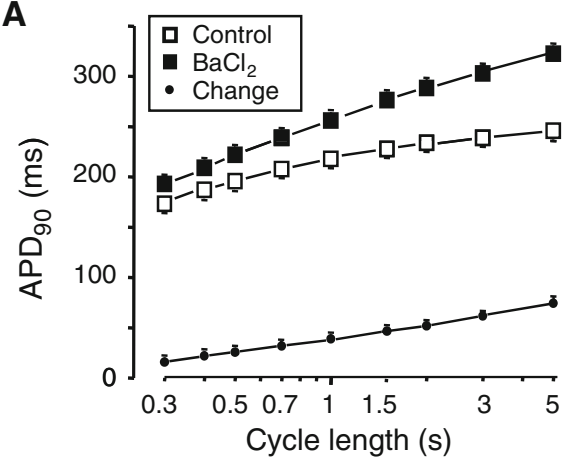

C

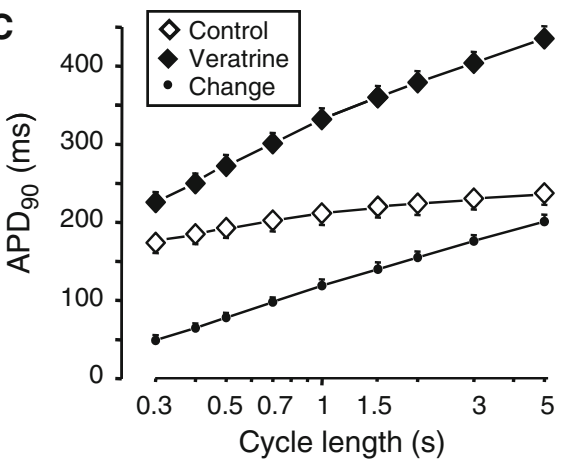

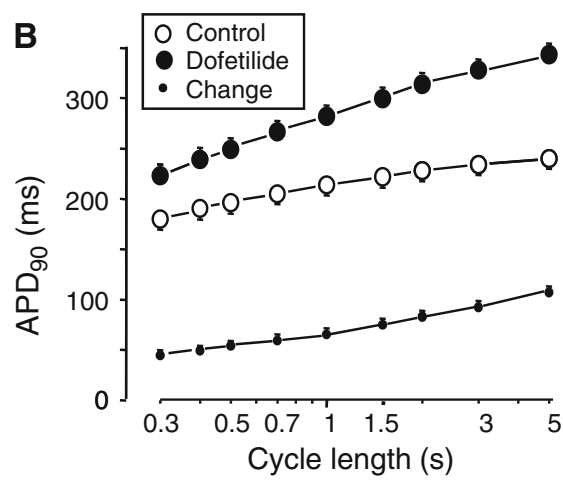

D

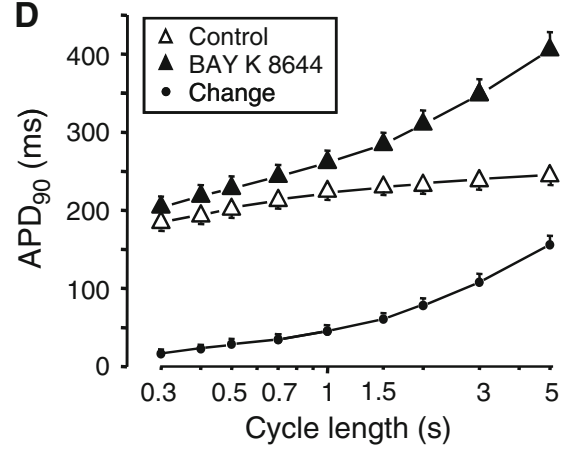

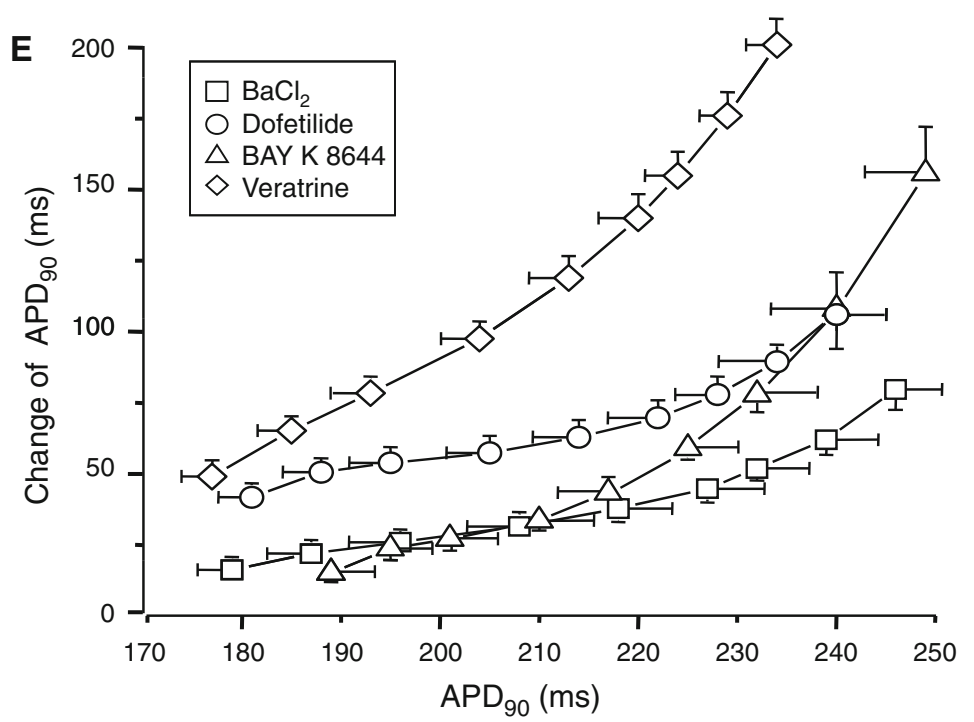


Table 1 Drugs used to modify action potential duration

\begin{tabular}{|c|c|c|c|}
\hline Drug & Concentration & Action & $\begin{array}{l}\text { Expected } \\
\text { change in APD }\end{array}$ \\
\hline Dofetilide & $50 \mathrm{nM}, 1 \mu \mathrm{M}^{\mathrm{a}}$ & Block of $I_{\mathrm{Kr}}$ & Lengthening \\
\hline Sotalol & $20-40 \mu \mathrm{M}^{\mathrm{b}}$ & Block of $I_{\mathrm{Kr}}$ & Lengthening \\
\hline E-4031 & $1 \mu \mathrm{M}$ & Block of $I_{\mathrm{Kr}}$ & Lengthening \\
\hline $\mathrm{BaCl}_{2}$ & $10 \mu \mathrm{M}$ & Block of $I_{\mathrm{K} 1}$ & Lengthening \\
\hline Veratrine & $1 \mu \mathrm{g} / \mathrm{ml}$ & Enhancement of $I_{\mathrm{Na}}$ & Lengthening \\
\hline BAY K 8644 & $1 \mu \mathrm{M}$ & Enhancement of $I_{\mathrm{Ca}}$ & Lengthening \\
\hline Mexiletine & $10 \mu \mathrm{M}$ & Block of $I_{\mathrm{Na}}$ & Shortening \\
\hline Tetrodotoxin & $2 \mu \mathrm{M}$ & Block of $\underline{I}_{\mathrm{Na}}$ & Shortening \\
\hline Lemakalim & $15 \mu \mathrm{M}$ & $\begin{array}{l}\text { Enhancement } \\
\text { of } I_{\mathrm{K}(\mathrm{ATP})}\end{array}$ & Shortening \\
\hline
\end{tabular}

a $50 \mathrm{nM}$ in human and $1 \mu \mathrm{M}$ in canine papillary muscles

b 20,30 , and $40 \mu \mathrm{M}$ in rabbit, human, and guinea pig papillary muscles, respectively

\section{Results}

RRD in human and canine myocardium

The rate-dependent effects of several cardioactive compounds were tested on APD in human (dofetilide, sotalol, E-4031, $\mathrm{BaCl}_{2}$, mexiletine, and TTX) and canine (dofetilide, $\mathrm{BaCl}_{2}$, veratrine, and Bay $\mathrm{K}$ 8644) multicellular ventricular preparations (Figs. 1, 2). The compounds used to modulate repolarization were chosen to either prolong or shorten APD based on modulation of different ion channels, as summarized in Table 1. Although the magnitude of APD changes varied with the applied compound, both the shortening and lengthening effects showed clear-cut RRD (i.e., the effects were larger at longer than at shorter CLs) with all the compounds tested (Figs. 1a-f, 2a-d).

Dependency of drug-induced APD changes on the pre-drug baseline APD is shown in Figs. 1g, h, 2e. In both species druginduced changes of APD were proportional to baseline APD. The relationship tended to become steeper at larger APD values. This property was differently expressed with different drugs and was most obvious for BAY K 8644 (Fig. 2e).

RRD in guinea pig and rabbit myocardium

The coincidence observed between human and canine ventricular tissues is not surprising, because the electrophysiological properties of human and canine myocardium are believed to be quite similar [8, 13, 14]. In contrast, the type and density of ion channels underlying the action potential in guinea pig and rabbit hearts are markedly different from those found in dog and human [15]. Furthermore, as opposed to the other species tested, APD displayed a non-monotonic dependency on CL in rabbits, maximum APD being achieved at an intermediate CL between 0.5 and $0.7 \mathrm{~s}$. This provided a unique opportunity to discriminate between rate-dependency and APDdependency of drug-induced APD changes. In this case, sotalol and lemakalim were used to prolong and shorten APD, respectively.

As expected, sotalol lengthened and lemakalim shortened APD in papillary muscles of both species (Fig. 3a-d). In guinea pig, similar to results obtained in canine and human preparations, both APD lengthening and shortening displayed monotonic RRD. In rabbit preparations the maximal drug-induced APD changes occurred at an intermediate CL between 0.5 and $0.7 \mathrm{~s}$, at which baseline APD was the longest (Fig. 3e, f). This suggests that the magnitude of drug-effect depended on baseline APD, rather than on pacing rate. This interpretation was supported by the analysis presented in Fig. 4, which shows that the druginduced APD changes were, in both species, related to baseline APD. Moreover, when the APD-CL relationship was non-monotonic in rabbit, similar APDs occurring at different CLs were associated with the same magnitude of APD changes. These observations lead us to conclude that longer action potentials are more sensitive to modulation than shorter ones, irrespective of the actual pacing rate.

\section{APD modulation by current injection}

Independency of RRD from the features (rate-dependency, profile, etc.) of the modulated current was tested by changing APD through the injection of a constant current. For technical reasons, these experiments were performed in single myocytes.

Inward $(-30 \mathrm{pA})$ and outward $(+60 \mathrm{pA})$ current pulses were injected throughout repolarization to lengthen and shorten APD, respectively, in isolated canine ventricular cells. As shown in Fig. 5a, b, the effect of current injection on APD was reversely rate-dependent. Furthermore, the changes in APD induced by the inward and outward current pulses were linearly proportional to the baseline APD values measured prior to current injection (Fig. 5c). In another series of experiments APD was lengthened or shortened by injections of current pulses of various amplitudes (ranging from -40 to $+100 \mathrm{pA}$ ) and the lengthening effect of $\mathrm{BaCl}_{2}$ was tested. $\mathrm{BaCl}_{2}$ induced greater APD lengthening when the pre-drug APD was longer, in spite of the constant pacing CL of $1 \mathrm{~s}$ applied in these experiments (Fig. 6).

Constancy of the relationship between APD and $I_{\text {net }}$

The assumption that APD modulation reflects a change in $I_{\text {net }}$, independently of the specific current affected by the drug, was verified by testing whether modulation of 
Fig. 3 Cycle length-dependent changes in APD induced by sotalol and lemakalim in guinea pig and rabbit ventricular preparations. a, b Effect of $40 \mu \mathrm{M}$ sotalol $15 \mu \mathrm{M}$ lemakalim in guinea pig papillary muscles $(n=5$ and $n=11$, respectively).

c, d Effect of $20 \mu \mathrm{M}$ sotalol $15 \mu \mathrm{M}$ lemakalim in rabbit papillary muscles $(n=5$ and $n=13$, respectively). Druginduced APD changes are presented in panels $\mathbf{e}$ and $\mathbf{f}$. Symbols and bars denote means \pm SEM
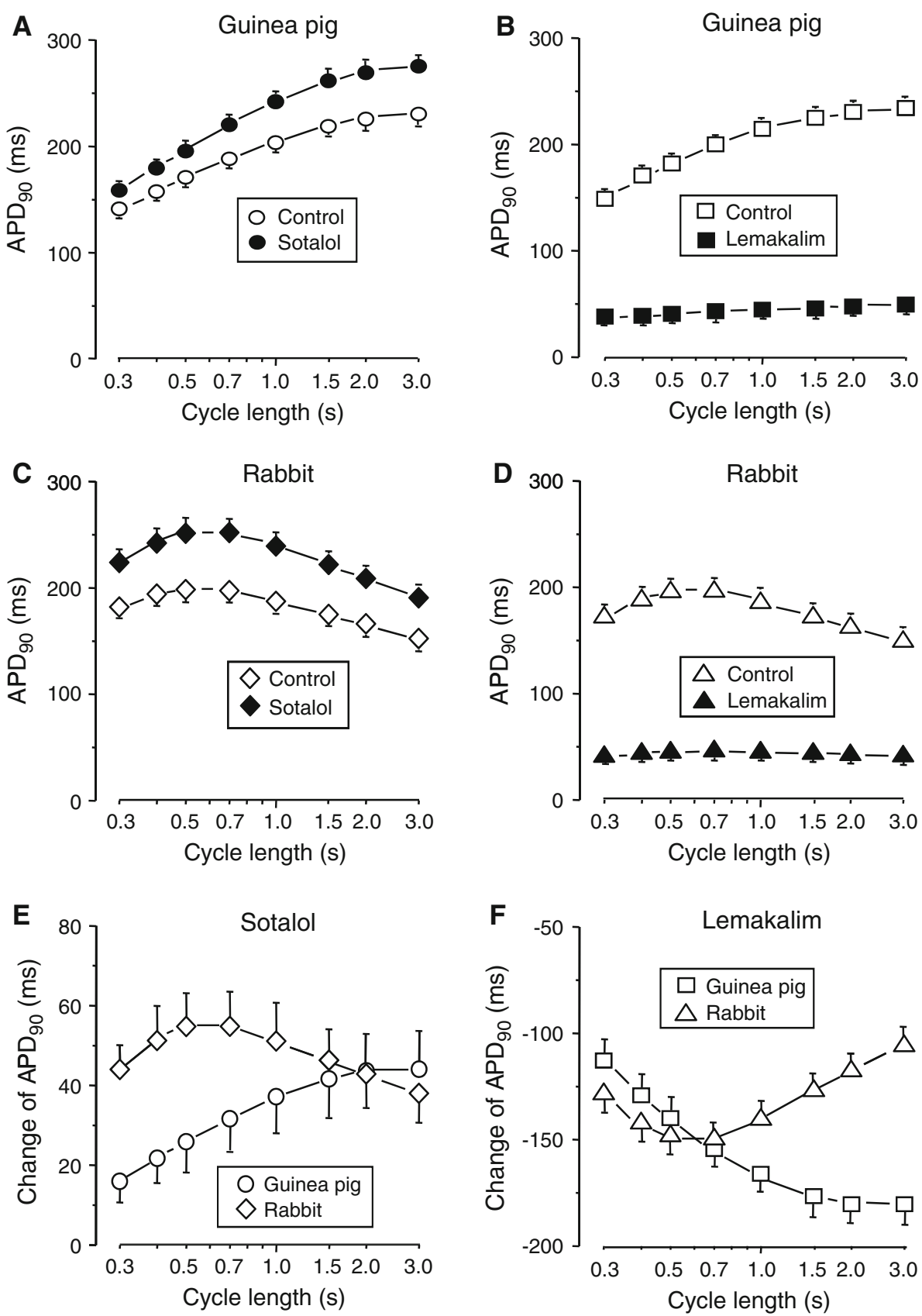

different currents led to changes in the relationship between APD and $I_{\text {net }}$ of human papillary muscles. To this end, APD was plotted as a function of $I_{\text {net }}$, measured at mid repolarization (see "Methods") in control and in the presence of various drugs. As shown in Fig. 7, APD was a non-linear function of $I_{\text {net }}$, and the $I_{\text {net }}$-APD relationships obtained in the presence of different drugs closely overlapped.

\section{Discussion}

The findings of this study indicate that (1) RRD is a general property of APD modulation, equally represented in different species, including dog, rabbit, guinea pig, and man; (2) the extent of drug-induced APD change is a function of baseline APD, irrespective of pacing rate; (3) the shape of the function relating APD to $I_{\text {net }}$, measured at an arbitrary point during repolarization, was substantially independent of the drug used to modulate APD.

RRD was recently interpreted as the consequence of the numerical relationship between APD and $I_{\text {net }}$ (proportional to repolarization velocity) $[1,20]$. As such, RRD should be seen as the indirect consequence of an intrinsic property of APD modulation, conserved across different species and types of modulatory interventions. Such an interpretation was supported by numerical modeling, which was based on 
Fig. 4 APD changes induced by sotalol (a, b) and lemakalim $(\mathbf{c}, \mathbf{d})$ in guinea pig $(\mathbf{a}, \mathbf{c})$ and rabbit $(\mathbf{b}, \mathbf{d})$ papillary muscles plotted against the respective baseline (pre-drug) APD values. Data are derived from experiments shown in Fig. 3 . Symbols and bars denote means \pm SEM
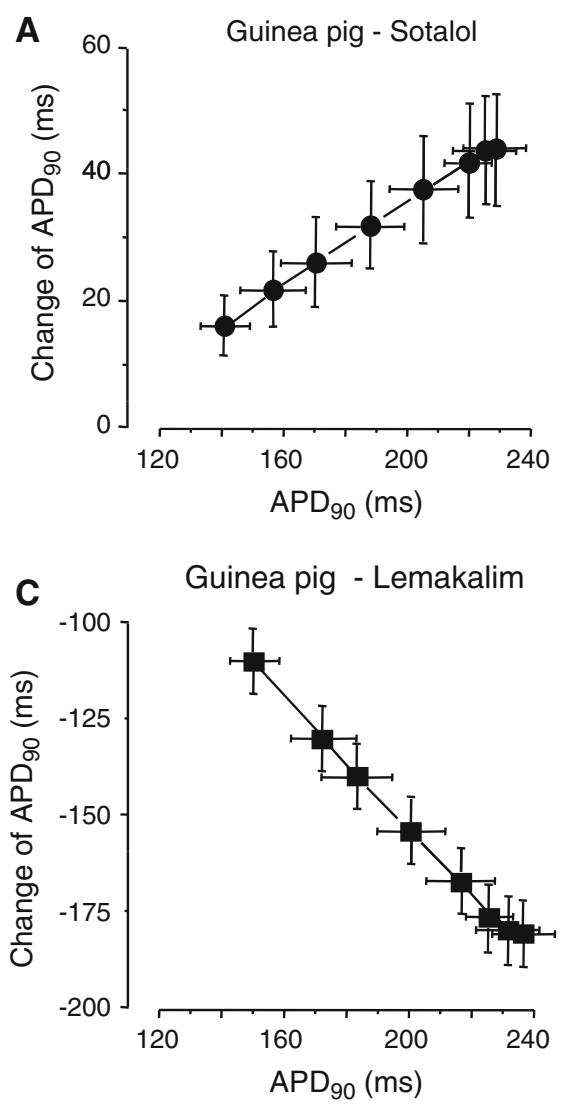
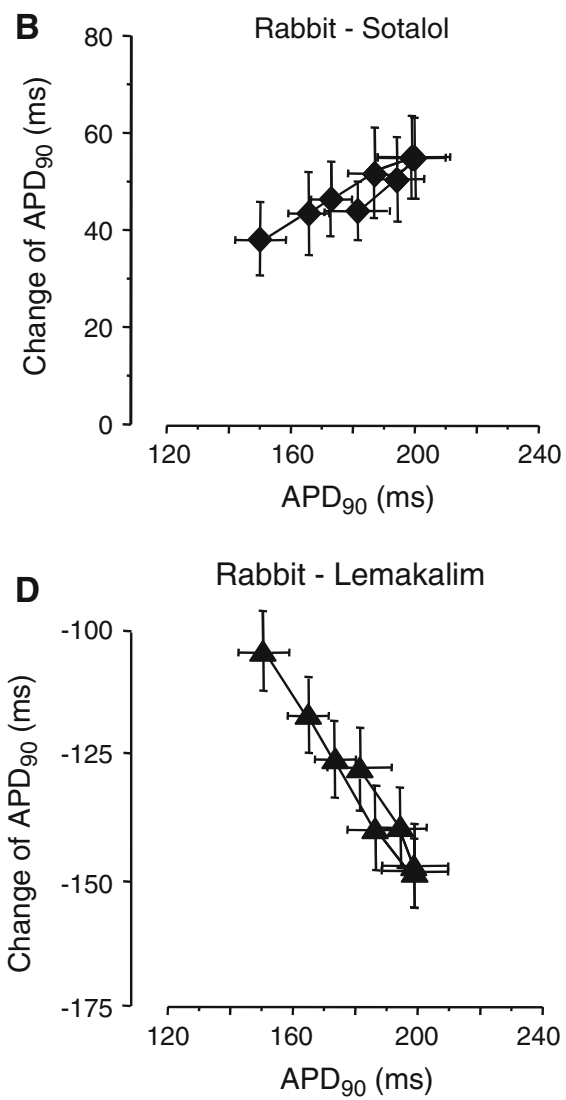

an assumption and led to the predictions tested in this study (see "Introduction"). In spite of a similar action potential contour, repolarization mechanisms significantly differ between larger mammals (dog, man) and smaller ones (rabbit, guinea pig). The observation that RRD is a general feature across these species supports the view that it may be accounted for by a general property of the system. If RRD depended on changes in the proportion of individual currents expressed at each cycle length, rather than on changes in $I_{\text {net }}$, the relationship between APD and $I_{\text {net }}$ should differ among drugs acting on different ion channels. APD- $I_{\text {net }}$ relationships obtained with different drugs largely overlapped instead, thus confirming that $I_{\text {net }}$ determines APD, independently of which channel is modulated. This validates the assumption underlying our RRD interpretation.

Finally, drug-induced APD changes proved to be directly determined by baseline APD, instead of the pacing rate. This confirms the prediction that APD modulation by drugs is intrinsically more pronounced under conditions when action potentials are ab ovo longer, with pacing rate being just one factor causing baseline APD to change. Although the view that the mathematical relationship between APD and $I_{\text {net }}$ may cause RRD is fully confirmed by the present results, several observations indicate that additional mechanisms may account for the extent to which
RRD is expressed. For instance, if the same mechanism accounted for RRD in all cases, we would expect the relationship between drug-induced APD changes and baseline APD to be identical in all cases. However, such a relationship was almost linear with some drugs (e.g. Fig. 4), but non-linear with others (e.g. Fig. 2e). This implies that even the dependency of APD modulation on baseline APD may include components that cannot be explained by a single mechanism. Furthermore, the existence of an intrinsic dependency of APD modulation on baseline APD does not rule out that other genuinely ratedependent mechanisms, such as drug-ion channel interactions $[5,9,19]$ and rate dependency of ion current kinetics $[3,12,16]$, may contribute to determine the magnitude of RRD. Indeed, whereas the same extent of RRD would be expected if only the "intrinsic" mechanism was operative, the magnitude of RRD was different between the various drugs tested.

Good example for RRD resulting from rate dependency of ion current kinetics was observed in murine ventricular myocardium exposed to sotalol [3]. In that study $20 \mu \mathrm{M}$ sotalol prolonged APD with reverse rate dependency. Since in the same preparation APD was not rate-dependent under baseline conditions within the relatively narrow range of the studied frequencies [3, 17], reverse rate-dependency cannot be accounted for the intrinsic mechanism described 

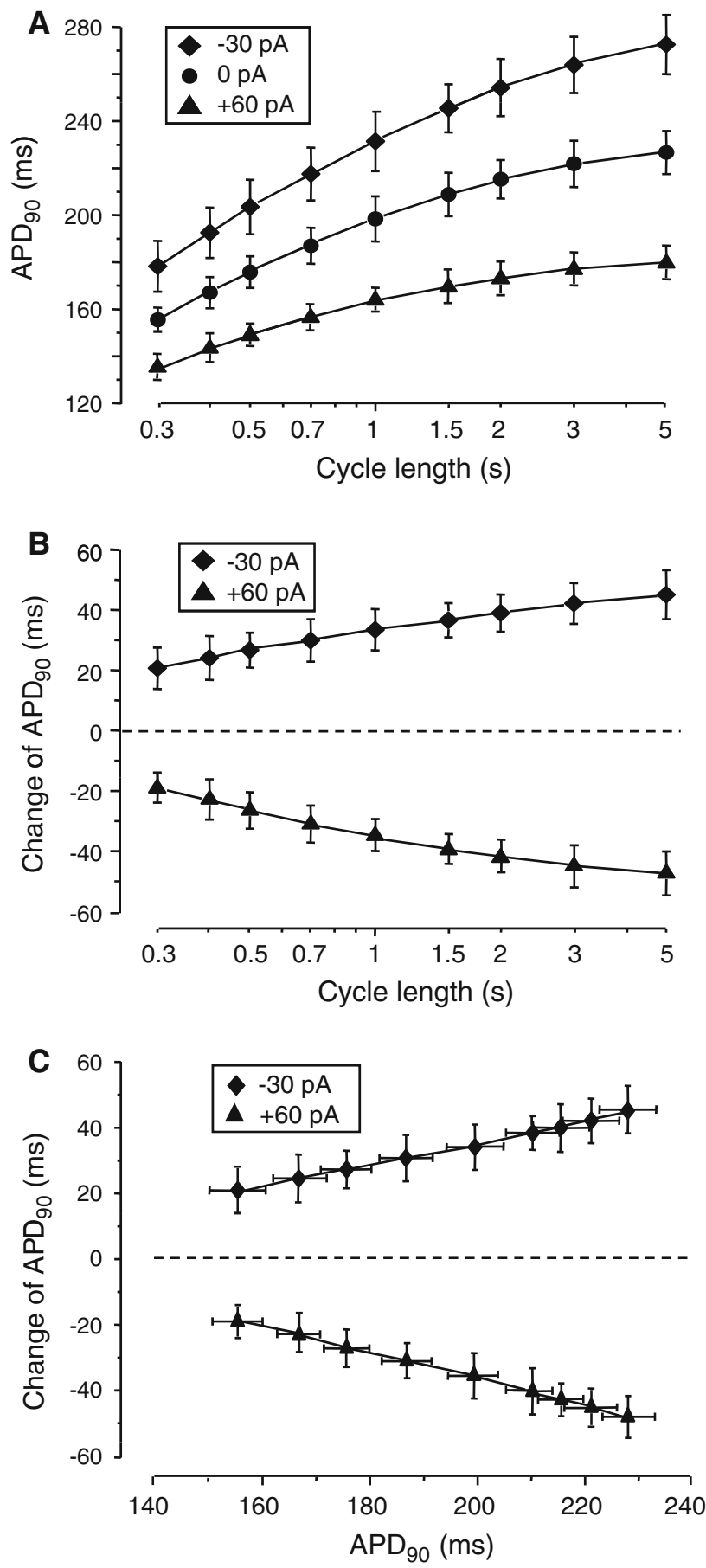

Fig. 5 Cycle length-dependent changes in APD induced by inward $(-30 \mathrm{pA})$ and outward $(+60 \mathrm{pA})$ current pulses, injected throughout the entire duration of the action potential, in single canine ventricular cells $(n=10)$. a Measured APD $_{90}$ values. b Changes in APD induced by the current pulses. c APD changes plotted against the control $(0 \mathrm{pA})$ APD values. Symbols and bars denote means \pm SEM

above. Consequently, an additional mechanism, very likely an $I_{\text {to }}$ blockade, must be responsible for the sotalol-induced RRD in mice. In sheep Purkinje fibers $10 \mu \mathrm{M}$ sotalol suppressed significantly (by 23\%) $I_{\text {to }}$, a current whose
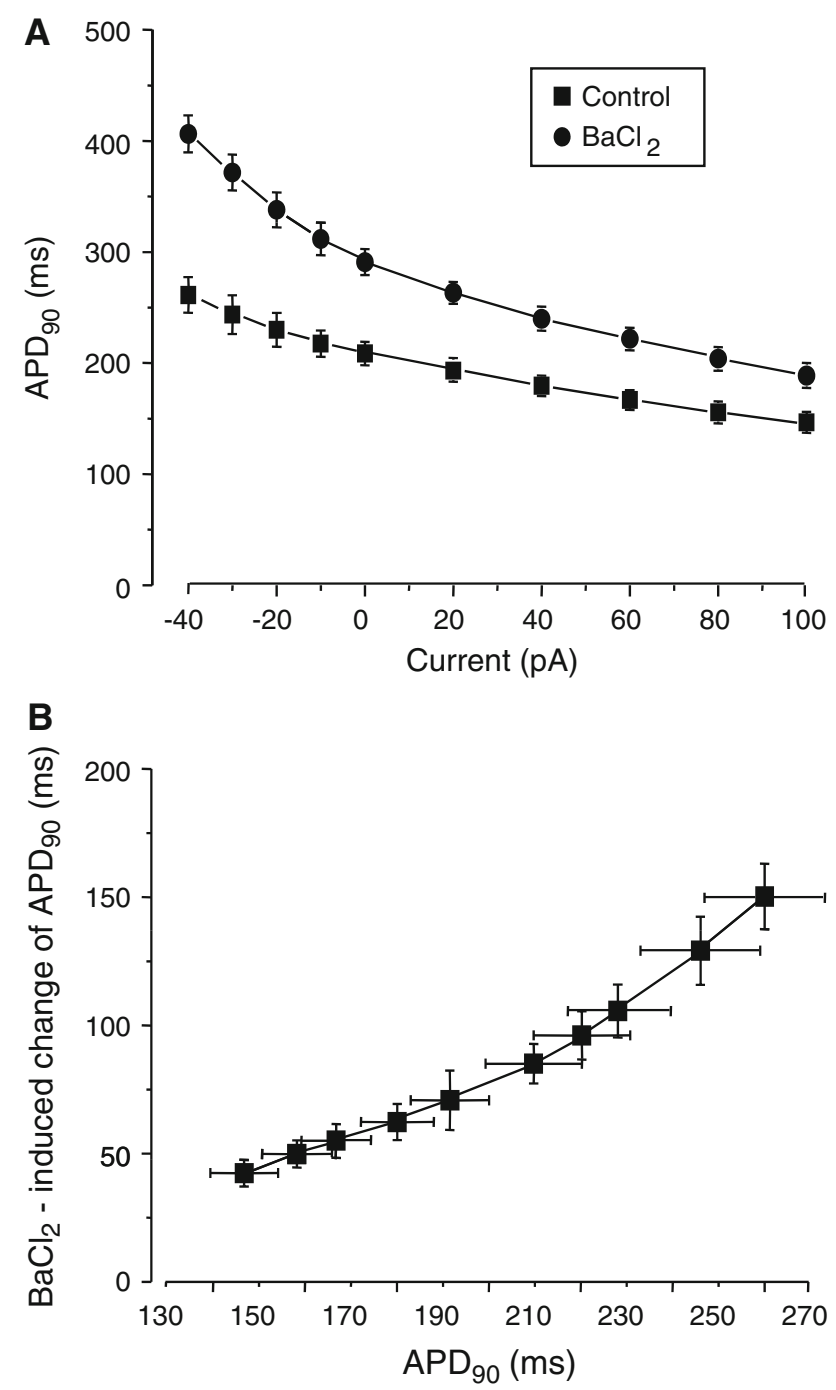

Fig. 6 Effect of $3 \mu \mathrm{M} \mathrm{BaCl} 2$ on isolated canine myocytes $(n=7)$ paced at a constant CL of $1 \mathrm{~s}$. APD was lengthened or shortened by current pulses of various amplitudes (ranging from -40 to $+100 \mathrm{pA}$ ) in the absence (control) and presence of $\mathrm{BaCl}_{2}$. The APD lengthening effect of $\mathrm{BaCl}_{2}$ was plotted as a function of the injected current (a) or against the control (pre-drug) APD values (b). Symbols and bars denote means \pm SEM

amplitude is known to decrease at short CLs due to incomplete recovery from inactivation [2]. Since $I_{\text {to }}$ is a strong repolarizing current in murine myocardium, and $I_{\text {to }}$ is expectedly larger at longer CLs, its block can reduce $I_{\text {net }}$ to a larger extent at slow rates.

\section{Conclusions}

The extent by which APD is changed by interventions depends on the initial APD, irrespective of rate. Since the initial APD is generally longer at lower rates, this may 


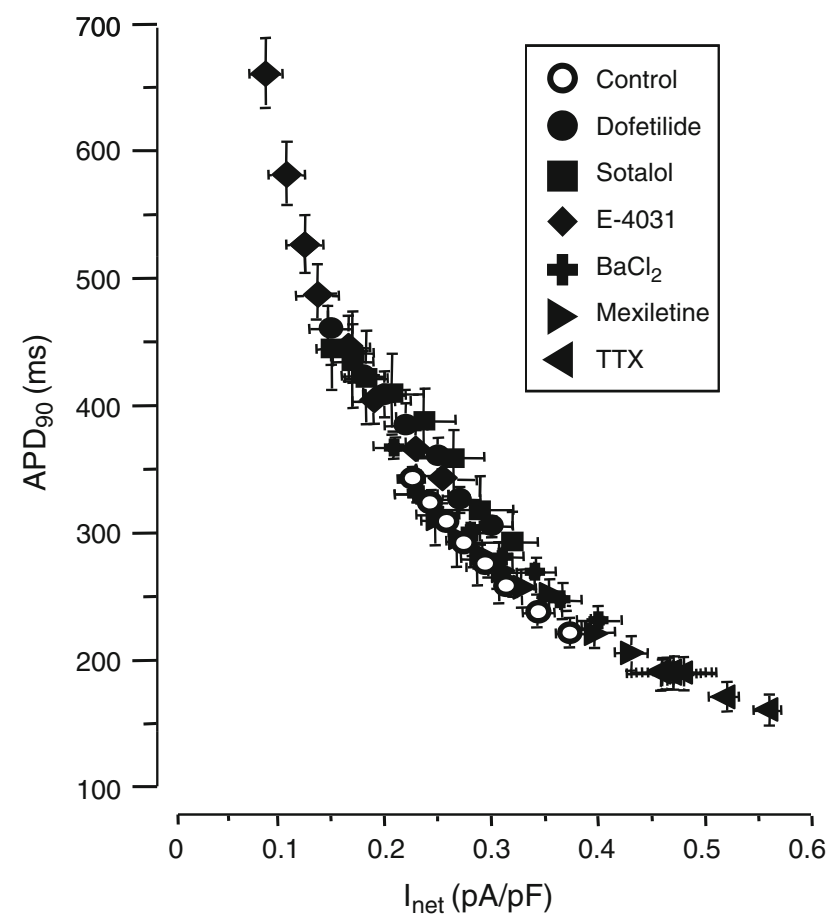

Fig. 7 Action potential duration $\left(\mathrm{APD}_{90}\right)$ plotted as a function of the net membrane current $\left(I_{\text {net }}\right)$ measured at half duration of the action potential. $I_{\text {net }}$ was determined from the slope of membrane potential changes $(\mathrm{d} V / \mathrm{d} t)$. Data are obtained from experiments performed in human papillary muscles paced at CLs varying between 0.3 and $5 \mathrm{~s}$, as shown in Fig. 1. Symbols and bars denote means \pm SEM

explain why RRD is so common and supports its origin from an "intrinsic" property independent of the modulation mechanism [1, 17]. Nevertheless, properties of the affected current and genuinely rate-dependent changes in its kinetics may overlap with intrinsic RRD and modulate its magnitude in a drug-specific way.

Acknowledgements This work was supported by grants from the Hungarian Scientific Research Fund (K-68457, K-73160, CNK-77855, F-67879, T-68911), the National Office for Research and Technology, National Technology Programme (TECH_08_A1_CARDIO08), European Community (EU FP7 grant ICT-2008224381, preDiCT), and the Hungarian Academy of Sciences. Further support was obtained from the Medical and Health Science Center of University of Debrecen (MEC-14/2008) and from the János Bolyai Research Scholarship (N.J.)

\section{References}

1. Bányász T, Horváth $B$, Virág L, Bárándi L, Szentandrássy $N$, Harmati G, Magyar J, Marangoni S, Zaza A, Varró A, Nánási PP (2009) Reverse rate dependency is an intrinsic property of canine cardiac preparations. Cardiovasc Res 84:237-244

2. Berger F, Borchard U, Hafner D (1989) Effects of (+)- and ( \pm )sotalol on repolarizing outward currents and pacemaker current in sheep cardiac Purkinje fibres. Naunyn-Schmiedeberg's Arch Pharmacol 340:696-704
3. Fabritz L, Kirchhof P, Franz MR, Eckardt L, Mönnig G, Milberg P, Breithardt G, Haverkamp W (2003) Prolonged action potential durations, increased dispersion of repolarization, and polymorphic ventricular tachycardia in a mouse model of proarrhythmia. Basic Res Cardiol 98:25-32

4. Hondeghem LM, Katzung BG (1977) Time- and voltage-dependent interactions of antiarrhythmic drugs with cardiac sodium channels. Biochim Biophys Acta 472:373-398

5. Hondeghem LM, Katzung BG (1984) Antiarrhythmic agents: the modulated receptor mechanism of action of sodium and calcium channel-blocking drugs. Ann Rev Pharmacol Toxicol 24:387-423

6. Hondeghem LM, Snyders DJ (1990) Class III antiarrhythmic agents have a lot of potential but a long way to go. Reduced effectiveness and dangers of reverse use dependence. Circulation 81:686-690

7. Horváth B, Magyar J, Szentandrássy N, Birinyi P, Nánási PP, Bányász T (2006) Contribution of $I_{\mathrm{Ks}}$ to ventricular repolarization in canine myocytes. Pflügers Arch 452:698-706

8. Jost $\mathrm{N}$, Acsai K, Horváth $\mathrm{B}$, Bányász T, Bitay $\mathrm{M}$, Bogáts $\mathrm{G}$, Nánási PP (2009) Contribution of $\mathrm{I}_{\mathrm{Kr}}$ and $\mathrm{I}_{\mathrm{K} 1}$ to ventricular repolarization in canine and human myocytes. Is there any influence of action potential duration? Basic Res Cardiol 104:33-41

9. Jurkiewicz NK, Sanguinetti MC (1993) Rate-dependent prolongation of cardiac action potentials by a methanesulfonanilide class III antiarrhythmic agent. Specific block of rapidly activating delayed rectifier $\mathrm{K}^{+}$current by dofetilide. Circ Res 72:75-83

10. Magyar J, Bányász T, Szigligeti P, Körtvély Á, Jednákovits A, Nánási PP (2000) Electrophysiological effects of bimoclomol in canine ventricular myocytes. Naunyn Schmiedeberg's Arch Pharmacol 361:303-310

11. Nair LA, Grant AO (1997) Emerging class III antiarrhythmic agents: mechanism of action and proarrhythmic potential. Cardiovasc Drugs Ther 11:149-167

12. Rocchetti M, Besana A, Gurrola GB, Possani LD, Zaza A (2001) Rate dependency of delayed rectifier currents during the guineapig ventricular action potential. J Physiol 534:721-732

13. Szabó G, Szentandrássy N, Bíró T, Tóth IB, Czifra G, Magyar J, Bányász T, Varró A, Kovács L, Nánási PP (2005) Asymmetrical distribution of ion channels in canine and human left ventricular wall: epicardium versus midmyocardium. Pflügers Arch 450:307-316

14. Szentadrássy N, Bányász T, Bíró T, Szabó G, Tóth B, Magyar J, Lázár J, Varró A, Kovács L, Nánási PP (2005) Apico-basal inhomogeneity in distribution of ion channels in canine and human ventricular myocardium. Cardiovasc Res 65:851-860

15. Varró A, Lathrop DA, Hester SB, Nánási PP, Papp JGY (1993) Ionic currents and action potentials in rabbit, rat and guinea pig ventricular myocytes. Basic Res Cardiol 88:93-102

16. Virág L, Acsai K, Hála O, Zaza A, Bitay M, Bogáts G, Papp JG, Varró A (2009) Self augmentation of the lengthening of repolarization is related to the shape of the cardiac action potential: implication for reverse rate dependency. $\mathrm{Br} \mathrm{J}$ Pharmacol 156:1076-1084

17. Waldeyer C, Fabritz L, Fortmueller L, Gerss J, Damke D, Blana A, Laakmann S, Kreinkamp N, Volkery D, Breithardt G, Kirchhof P (2009) Regional, age-dependent, and genotypedependent differences in ventricular action potential duration and activation time in 410 Langendorff-perfused mouse hearts. Basic Res Cardiol 104:523-533

18. Weirich J, Antoni H (1998) Rate-dependence of antiarrhythmic and proarrhythmic properties of class I and class III antiarrhythmic drugs. Basic Res Cardiol 93(Suppl 1):125-132

19. Yang T, Roden DM (1996) Extracellular potassium modulation of drug block of $\mathrm{I}_{\mathrm{Kr}}$. Implications for torsade de pointes and reverse use-dependence. Circulation 93:407-411

20. Zaza A (2009) Control of cardiac action potential: the role of repolarization dynamics. J Mol Cell Cardiol. doi:10.1016/j. yjmcc.2009.07.027. (in press) 